\title{
ARTICLE
}

Received 22 Jan 2015 | Accepted 25 Mar 2015 | Published 11 May 2015

DOI: 10.1038 /ncomms 8024

\section{RNA structure determination by solid-state NMR spectroscopy}

\author{
Alexander Marchanka', Bernd Simon, Gerhard Althoff-Ospelt ${ }^{2} \&$ Teresa Carlomagno 1,3
}

Knowledge of the RNA three-dimensional structure, either in isolation or as part of RNP complexes, is fundamental to understand the mechanism of numerous cellular processes. Because of its flexibility, RNA represents a challenge for crystallization, while the large size of cellular complexes brings solution-state NMR to its limits. Here, we demonstrate an alternative approach on the basis of solid-state NMR spectroscopy. We develop a suite of experiments and RNA labeling schemes and demonstrate for the first time that ssNMR can yield a RNA structure at high-resolution. This methodology allows structural analysis of segmentally labelled RNA stretches in high-molecular weight cellular machines-independent of their ability to crystallize - and opens the way to mechanistic studies of currently difficultto-access RNA-protein assemblies.

\footnotetext{
${ }^{1}$ Structural and Computational Biology Unit, European Molecular Biology Laboratory, Meyerhofstrasse 1, 69117 Heidelberg, Germany. ${ }^{2}$ Bruker BioSpin, Silberstreifen 4, 76287 Rheinstetten, Germany. ${ }^{3}$ Helmholtz Zentrum für Infektionsforschung, Inhoffenstrasse 7, 38124 Braunschweig, Germany. Correspondence and requests for materials should be addressed to A.M. (email: marchank@embl.de) or to T.C. (email: teresa.carlomagno@embl.de).
} 
n gene expression regulation, stress response and pathogens infection, a multitude of non-coding RNAs and ribonucleoprotein complexes accomplish their function cycling through transient intermolecular contacts and related conformational changes. Taking influence on these processes requires a mechanistic understanding of the intermolecular interactions, which, in turn, necessitates structural information. Both naked RNAs and RNPs represent a challenge for structural biology. The conformational plasticity of the RNA restricts application of $\mathrm{X}$-ray crystallography, while the high-molecular weight of the RNA (or RNP) of interest pushes solution-state NMR to its limits. Lately, solid-state NMR (ssNMR) spectroscopy, which is applicable to macromolecules of any size in non-crystalline form, has emerged as a powerful alternative to study the structure of amyloid fibrils ${ }^{1,2}$, membrane proteins ${ }^{3,4}$, and large proteinprotein assemblies ${ }^{5}$. Despite these successes, ssNMR has been rarely applied to nucleic acids, and the methodology for RNA structure determination is still lacking ${ }^{6}$. Here, we present the first de novo structure determination of RNA by ssNMR, together with the experimental methods we developed for it. We demonstrate that RNA structure is accessible at high resolution by ssNMR using a few, easy to prepare, nucleotide-type selectively labeled samples. This methodology opens the way to the structure of RNA stretches in large RNA-protein assemblies, independent of their ability to crystallize, and thus to mechanistic studies of yet inaccessible cellular machines.

\section{Results}

The Box C/D RNA bound to L7Ae. In this study, we solve the structure of the 26mer Box C/D RNA from Pyrococcus Furiosus $(P f)$ as part of the complex with the protein L7Ae (Fig. 1a and
Supplementary Fig. 1 ). ${ }^{13} \mathrm{C}$ and ${ }^{15} \mathrm{~N}$ line widths of $0.4-0.5$ and $0.6-0.8$ p.p.m., respectively, allow determining the structure by ssNMR data with a precision of $0.8 \AA$. We choose to study this RNA for the following reasons. First, we were unable to crystallize the L7Ae-Box C/D RNA complex with the RNA sequence of Fig. 1a, despite the existence of crystallographic structures of homologues ${ }^{7,8}$. This demonstrates that the crystallization of RNA-protein complexes can be unexpectedly challenging, in dependence of the RNA sequence. Second, the RNA of Fig. 1a contains the conserved Box $\mathrm{C}$ and Box D sequences, which build the so-called k-turn motif. The geometry of the k-turn is measured by an angle $\phi^{9}$, which is variable in the free RNA and depends on the concentration of magnesium ${ }^{10,11}$. Upon protein binding, the $\mathrm{k}$-turn parameter $\phi$ adopts a value close to $23^{\circ}$ for all k-turn motifs investigated to date, independent of the experimental method, the exact sequence of the RNA or the species it belongs to ref. 12. The conservation of this structural motif offers the opportunity to verify the accuracy of the structure obtained by ssNMR, beyond the differences to crystallographic reference structures expected as a consequence of packing forces and RNA-RNA contacts in the crystals.

Resonance assignment and measurement of distance restraints are the key steps in structure determination by ssNMR. In contrast to proteins, where homonuclear ${ }^{13} \mathrm{C},{ }^{13} \mathrm{C}$ correlations are sufficient for resonance assignment, the poor chemical shift dispersion of ribose resonances in RNA requires additional heteronuclear editing. We find that three-dimensional pulse schemes yield low signal-to-noise within our experimental set-up, while the quality of two-dimensional spectra allows for both assignment and quantification of cross-peaks. Therefore, our strategy does mainly without three-dimensional experiments and
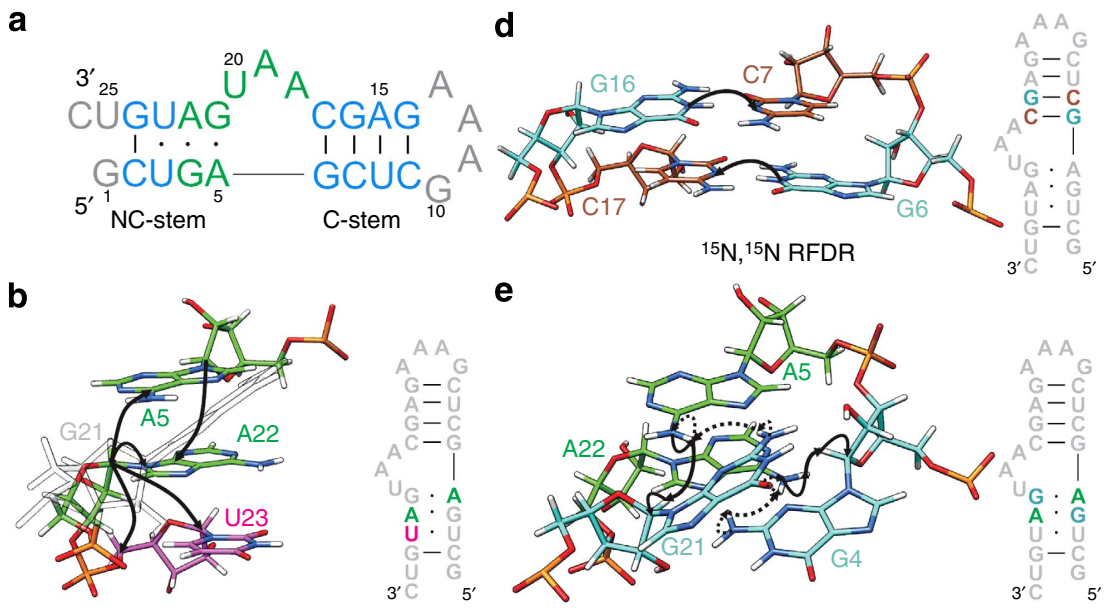

${ }^{13} \mathrm{C},{ }^{15} \mathrm{~N}-$ TEDOR- ${ }^{13} \mathrm{C},{ }^{13} \mathrm{C}-\mathrm{PDSD}$

e
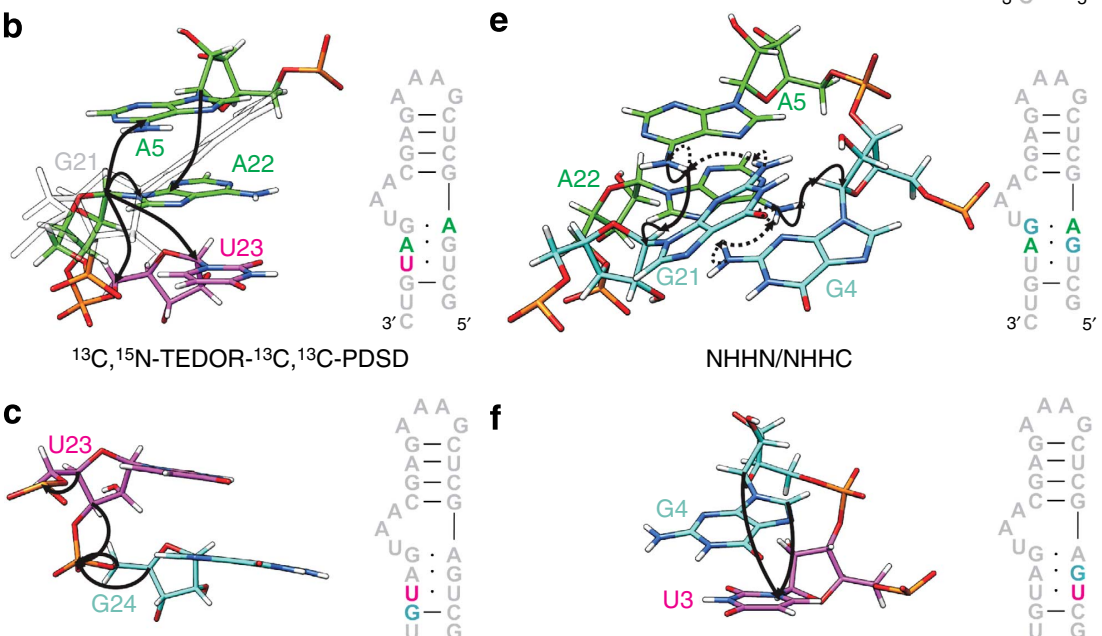

${ }^{13} \mathrm{C}-31 \mathrm{P}-\mathrm{TEDOR}$

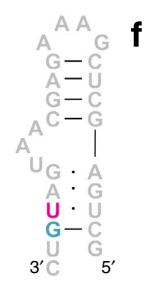

f

$\mathrm{NHHN} / \mathrm{NHHC}$
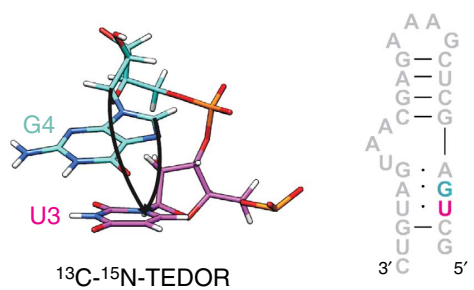

Figure 1 | Sequence of the Pf Box C/D RNA and magnetization transfer schemes. (a) Sequence and secondary structure of the Pf Box C/D RNA. Helical regions, light blue; k-turn, green; loop and termini, grey. (b-f) Schematic representation of the magnetization transfer schemes used for resonance assignment and distance measurement, shown on nucleotides stretches highlighted in the sequence. $A$, green; $G$, cyan; $C$, sienna; $U$, magenta.

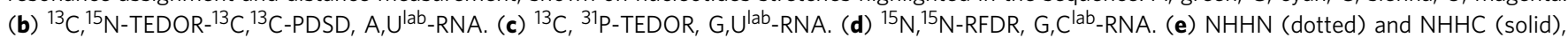
A, Glab-RNA. (f) ${ }^{13} \mathrm{C},{ }^{15} \mathrm{~N}-\mathrm{TEDOR},\left(\mathrm{G}-{ }^{13} \mathrm{C}, \mathrm{U}-{ }^{15} \mathrm{~N}\right){ }^{\text {lab }}-\mathrm{RNA}$. 
resolves spectral overlaps by selective labeling. To make the method accessible to a broad community, we abstain from using atom-selective labelling and employ only RNAs that can be produced with commercially available building blocks by in vitro transcription. In this study, we designed eight combinations of double-nucleotide-type selective-labelled RNAs (Supplementary Fig. 1), to accomplish both resonance assignment and measurement of structural parameters.

Sequence-specific assignment. First, we assigned the spinsystems of individual nucleotides in a non-site-specific manner ${ }^{13}$. For the 26mer Box C/D RNA, we found six adenosines, seven guanosines, three cytosines and four uridines spinsystems. Analysis of canonical coordinates of ribose shifts ${ }^{14}$ (Supplementary Fig. 2) suggests that two adenosines, three guanosines and one uridine are not located in regular A-form helices. This allowed us to attribute the uridine spin-system to U20, which was used as starting point for sequential assignment.

Seventeen out of the 26 nucleotides were assigned sitespecifically using correlations between the $\mathrm{C} 1$ ' or $\mathrm{C} 6 / \mathrm{C} 8$ atoms of nucleotide $i$ to the carbons of neighbouring nucleotides $i \pm 1$, as well as nucleotides of the opposite strand (Fig. 1b, Supplementary Table 1). To improve resolution, before the ${ }^{13} \mathrm{C},{ }^{13} \mathrm{C}$ transfer, the magnetization of $\mathrm{Cl}^{\prime}$ or $\mathrm{C} 6 / \mathrm{C} 8$ was correlated to the respective N1/N9 via TEDOR (Transferred-Echo-DOuble-Resonance) ${ }^{15}$, yielding a two-dimensional $(2 \mathrm{D}){ }^{15} \mathrm{~N},{ }^{13} \mathrm{C}$ correlation (Fig. 2a,b, Methods). This allows clear distinction of purine and pyrimidines in double-nucleotide-type selective-labelled samples (for example, $G, U^{l a b}$ - or A, $\left.U^{l a b}-R N A s\right)$. For the long-range carbon-carbon transfer, we tested different mixing sequences and finally settled on the PDSD (Proton-Driven-Spin-Diffusion) scheme due to its superior sensitivity ${ }^{16}$.
${ }^{13} \mathrm{C},{ }^{15} \mathrm{~N}-$ TEDOR- ${ }^{13} \mathrm{C},{ }^{13} \mathrm{C}-\mathrm{PDSD}$ (Supplementary Fig. 3a) was applied with a mixing time of $700 \mathrm{~ms}$ to six selective-labeled RNAs (Supplementary Fig. 1) and yielded several inter-nucleotide contacts up to a distance of 9-10 (Fig. 2a,b, Supplementary Figs 4 and 5). As an example of sequential assignment, G,Ulab - and A,U $U^{\text {lab }}$-RNAs yielded multiple correlations between the C1',C6 of U20 and both a guanosine and an adenosine spinsystem; the latter correlates further with another adenosine of the $A, U^{l a b}-R N A$. These cross-peaks are compatible with either an AAUG or a GUAA stretch, and identify unambiguously the spinsystem A18-A19-U20-G21. This strategy yielded sequential assignment of 17 out of 19 nucleotides in structured regions, excluding the tetra-loop and the terminal ends. The remaining two nucleotides (G14, A15) were assigned by substitution of the tetra-loop sequence GAAA with UUCG. This alleviated the overlap of G14 and A15 with the resonances of the GAAA loop, allowing their assignment, as well as the identification of G10 and of two adenosines of the A11-A13 stretch. The poor intensity of the GAAA tetra-loop resonances is indicative of conformational heterogeneity; likewise, the terminal G1, U25 and C26 spinsystems are not visible in any of the spectra and were not considered in the structure calculation.

Next, we tested the performance of a ${ }^{13} \mathrm{C},{ }^{31} \mathrm{P}$ correlation, which, with a TEDOR mixing time of $3.2 \mathrm{~ms}$, should provide sequential $C 2{ }_{i}^{\prime} / P_{i+1}$ and $C 3_{i}^{\prime} / P_{i+1}$ contacts. The mixing time was optimized for sensitivity of transfer over two to three bonds, ranging up to $4-5 \AA$ distance (Fig. 1c). As expected, the ${ }^{31} \mathrm{P}$ resonances are poorly resolved in helices and the spectrum provided information only for non A-form structural elements (Fig. 2c, Supplementary Fig. 6a,b).

Finally, we could sequentially assign $>90 \%$ of all carbon resonances of the Pf Box C/D RNA in the stretches 2-10 and $14-24$ (81\% for both carbons and nitrogens).
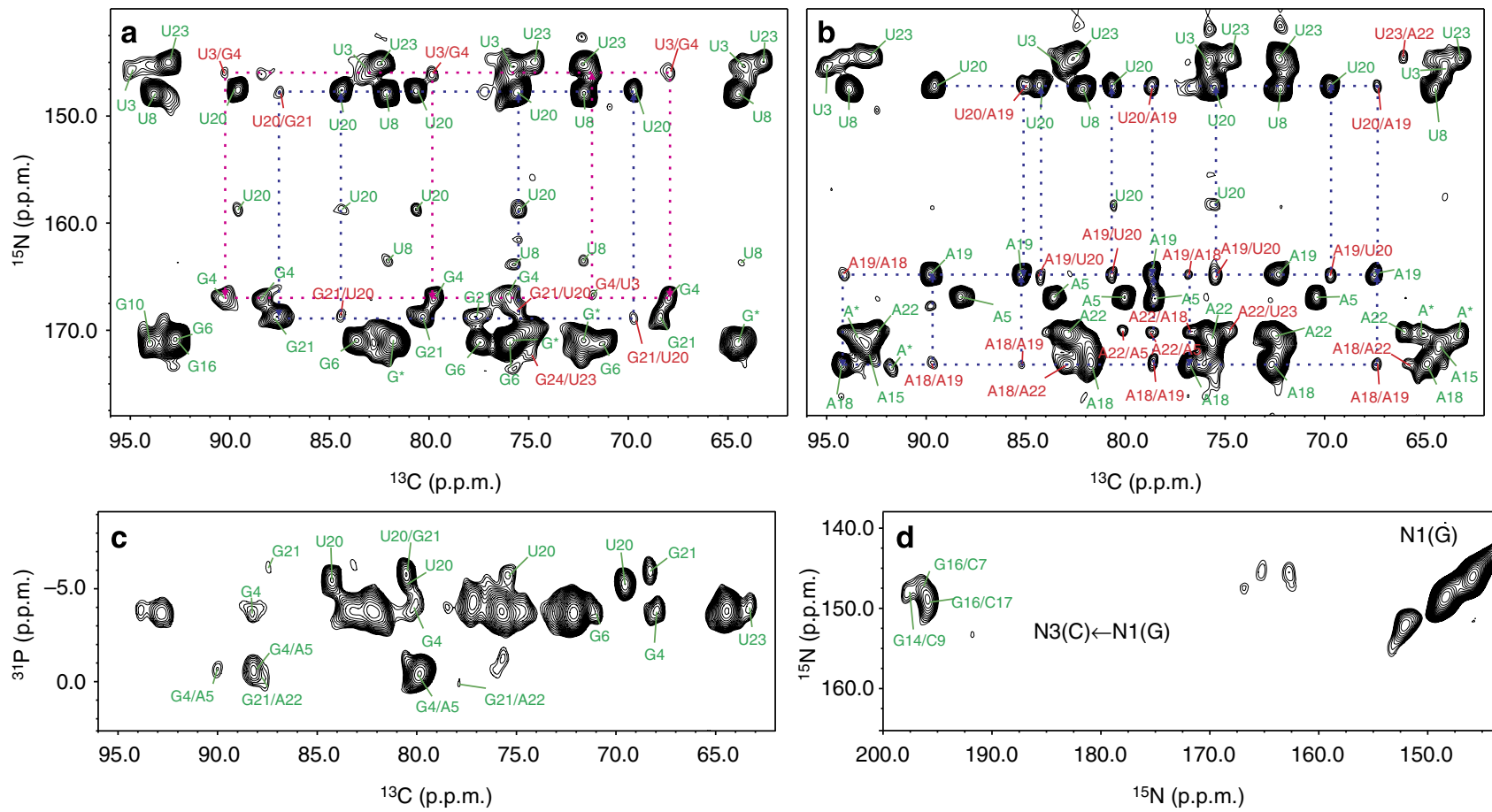

Figure 2 | ssNMR spectra for the sequential assignment and measurement of structural restraints. (a-b) Ribose region of $2 D{ }^{13} \mathrm{C},{ }^{15} \mathrm{~N}-\mathrm{TEDOR}-{ }^{13} \mathrm{C}$, ${ }^{13} \mathrm{C}$-PDSD spectra of (a), G, Ulab-RNA and (b) A, Ulab-RNA (mixing time, $700 \mathrm{~ms}$ ). Intra- and inter-nucleotide correlations are labeled in green and red, respectively. Selected sequential correlations are shown. Partially overlapped guanosines G10, G14, G16 are labeled as $\mathrm{G}^{\star}$; non-site-specifically assigned adenosines in the tetra-loop (A11-A13) are labeled as $A^{\star}$. (c) $2 D^{13} C_{1}^{31}$ P-TEDOR spectrum of $G, U^{\text {lab }}$-RNA. (d) $2 D{ }^{15} N_{1}^{15} \mathrm{~N}-R F D R$ spectrum showing the G-N1/C-N3 correlations for G:C base pairs. 
Structural determination of the RNA by ssNMR. The determination of RNA secondary structure requires the identification of base pairs. To this end, we used a ${ }^{15} \mathrm{~N},{ }^{15} \mathrm{~N}$ through-space correlation (RFDR, Radio-Frequency-DrivenRecoupling $)^{17,18}$ to reveal the spatial proximity of either A-N1 and U-N3 or G-N1 and C-N3 in Watson-Crick base pairs (Fig. 1d). The presence of three G:C base pairs (Fig. 2d) defined the C-stem. The G24:C2 base pair was not found due to the absence of the G24-N1 resonance in intra-nucleotide correlations, probably as a consequence of conformational heterogeneity at the helix ends.

Secondary structure prediction suggests one $U \cdot U$ and two A. G base pairs (Fig. 1a). Initially, to verify the presence and determine the topology of these non-canonical base pairs, we measured NHHN spectra ${ }^{19,20}$; in this experiment, magnetization is transferred between close-by ${ }^{15} \mathrm{~N}$ nuclei exploiting the spatial proximity of their attached protons (Fig. 1e). This strategy failed, due to severe overlap of the involved nitrogen resonances. Next, we recorded NHHC spectra (Fig. 1e) on three selectively labelled RNA samples (Supplementary Fig. 7); $(G, U)^{\text {lab }}$-RNA yielded weak $\mathrm{N} 2{ }_{\mathrm{G} 21} / \mathrm{Cl}_{\mathrm{G} 4}$ correlations, while $(\mathrm{A}, \mathrm{G})^{\text {lab }}$-RNA yielded strong $\mathrm{N6}_{\mathrm{A} 22} / \mathrm{Cl}^{\prime}{ }_{\mathrm{G} 4}$ and $\mathrm{N}_{\mathrm{A} 5} / \mathrm{Cl}_{\mathrm{G} 21}$ signals. The last two correlations were also detected in a ${ }^{13} \mathrm{C}$-band-selective, ${ }^{15} \mathrm{~N}$-TEDOR spectrum (Supplementary Fig. 6c). This pattern of cross-peaks, together with the anti conformation of the glycosidic angle $\chi$ for all four G and A nucleotides (vide infra), is exclusively compatible with two N7-amino, N3-imino base pairs, which are typical of k-turn motifs.

The U3 - U23 base pair might be detected from the proximity of the two $\mathrm{H} 3$ atoms in a NHHN correlation. In our case, the chemical shift difference of only 1 p.p.m. between the U3-N3 and U23-N3 hindered the resolution of the weak cross-peak from the intense diagonal. Therefore, we resorted to the analysis of chemical shifts (CS), as indicators of secondary structure. The CS of U23-C2 (151 p.p.m.) and both U3- and U23-C4 (165.6 and 167.6 p.p.m., respectively) deviate from the values of non-stacked disordered nucleotides (154.0 and 168.5 p.p.m.) as well as from the values of A-form helices (152.9 and 169.2 p.p.m.; ref. 21). The low CS of U23-C2 and U3-C4 indicate stacking on both sides, while for U3$\mathrm{C} 2$ and U23-C4 the up-field shift induced by stacking is compensated by the down-field shift of carbonyl acceptors of $\mathrm{H}$-bonds. All in all, CS analysis predicts that U3 and U23 form a U3 - U23 2-carbonyl-N3, 4-carbonyl-N3 base pair ${ }^{22}$.

Distance restraints. Next, we obtained distance restraints from four different correlation experiments: ${ }^{13} \mathrm{C},{ }^{15} \mathrm{~N}$-TEDOR- ${ }^{13} \mathrm{C}$, ${ }^{13} \mathrm{C}$-PDSD recorded at multiple mixing times provided carboncarbon distances; ${ }^{13} \mathrm{C},{ }^{31} \mathrm{P}-\mathrm{TEDOR}$ and ${ }^{13} \mathrm{C}$-band-selective, ${ }^{15} \mathrm{~N}$-TEDOR yielded a few carbon-phosphorus (17) and carbonnitrogen (6) distances, respectively; CHHC and NHHC experiments yielded distances between protons (Supplementary Table 2). In this context, we proved the applicability of more sophisticated and selective transfer schemes, such as PAR (Proton-Assisted-Recoupling) and PAIN (Proton-AssistedInsensitive-Nuclei) $)^{23,24}$. However, the sensitivity of these experiments remained too low, especially in combination with heteronuclear filtering.

The mixing sequence PDSD does not permit the quantitative measurement of distance restraints ${ }^{25,26}$; however, when recorded at multiple mixing times, it provided information on ( $\mathrm{Cl}^{\prime}, \mathrm{C} 8 /$ C6 $)_{i}-\left(\mathrm{C}_{x}\right)_{j}$ distance ranges. A total of 91 inter-nucleotide crosspeaks were obtained from the ${ }^{13} \mathrm{C},{ }^{15} \mathrm{~N}$-TEDOR- ${ }^{13} \mathrm{C},{ }^{13} \mathrm{C}-\mathrm{PDSD}$ experiments, which were all incorporated in structure calculations, in addition to 46 intra-nucleotide restraints over $\geq 3$ bonds (Supplementary Table 2 ).
Next, we attempted to obtain base-base $\mathrm{C}_{i}-\mathrm{N}_{i}{ }_{1}$ cross-peaks through a ${ }^{13} \mathrm{C}$-band-selective, ${ }^{15} \mathrm{~N}$-TEDOR experiment ${ }^{15}$ recorded for samples with ${ }^{13} \mathrm{C}$-labelling of one nucleotide type and ${ }^{15} \mathrm{~N}$-labelling of another nucleotide type (Fig. 1f). Our efforts were unsuccessful, due to low signal-to-noise. However, when recording a $\left({ }^{13} \mathrm{C} 1^{\prime},{ }^{13} \mathrm{C} 4\right)$-band-selective, ${ }^{15} \mathrm{~N}$-TEDOR, we obtained six inter-nucleotide cross-peaks from both the k-turn and helical regions (Supplementary Fig. 6c,d and Supplementary Table 2).

Finally, 2D NHHC and CHHC spectra ${ }^{19,27}$ yielded 17 and 10 inter-nucleotide contacts (Supplementary Figs 7 and 8), respectively, in addition to 21 intra-nucleotide correlations over $\geq 3$ bonds (Supplementary Table 2).

In addition to distance restraints, we obtained dihedral angles from analysis of ribose chemical shifts (Methods and Supplementary Fig. 2) and from CHHC experiments at short mixing times. Similarly to solution-state NMR, the $\chi$ angle was restrained to syn in the presence of a strong $\mathrm{C} 1$ '-C8/C6 crosspeak (short H1'-H8/H6 distance) and to anti in the other cases. Only A19 displayed a $\chi$ angle in the syn conformation, in agreement with other k-turn RNA structures ${ }^{11}$.

Structure calculations. Distance and dihedral angle restraints, as well as base pair restraints were used in ARIA $^{28}$ to calculate the structure of the Box C/D RNA from ssNMR data. Out of 300 calculated structures, the first 60 converged to a well-defined minimum with precision of $0.9 \AA$ (root-mean-square-deviation (r.m.s.d.) of all heavy atoms of nucleotides 2-9 and 14-24 of the first 20 structures; Fig. 3a and Table 1). The distances derived from the ${ }^{13} \mathrm{C},{ }^{15} \mathrm{~N}$-TEDOR- ${ }^{13} \mathrm{C},{ }^{13} \mathrm{C}$-PDSD spectra had the highest impact on the precision of the structure, followed by those derived from the $\mathrm{CHHC}$ and NHHC experiments (Supplementary Fig. 9). As for structural calculation from solution-state NMR data, the definition of the RNA secondary structure (topology of base pairs) was essential. The stem regions were defined by eight distance and nine angular restraints per residue, while the geometry of the k-turn required 21 distances per nucleotide. The structure determination method was validated by removal of random fractions of restraints. The structures bundles were consistent upon random removal of up to $20 \%$ of the total restraints.

\section{Discussion}

The 26mer Box C/D RNA used in this study does not crystallize in complex with L7Ae; however, the crystallographic structure of two orthologous complexes from Archaeoglobus fulgidus ${ }^{7,29}$ (Af, PDB code 1RLG and 4BW0), one orthologous complex from Solpholobus solfataricus ${ }^{30}$ (Ss, PDB code 3PLA) and another L7Ae-Box C/D RNA from $P f$ with a different RNA sequence ${ }^{8}$ (PDB code 3NMU) let us evaluate the accuracy of the ssNMR structure in the critical k-turn region (Fig. $3 \mathrm{~b}, \mathrm{c}$ ). The $\phi$ angle of $23^{\circ}$ that defines the k-turn geometry of the ssNMR structure is in very good agreement with the $\phi$ angles of the reference structures

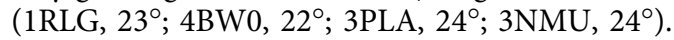

Next, we analysed the backbone and glycoside torsion angles of our structures bundle and compared them with the corresponding torsion angles of the four reference structures (Supplementary Fig. 10). We choose to compare torsion angles rather than r.m.s.d. values to better visualize the variability of both the crystallographic structures and our bundle at each nucleotide position. The $\delta$ torsion describes the ribose pucker and is defined by the chemical shift analysis of Supplementary Fig. 2. The values fit nicely to those of the reference structures, with the exception of 5 , 19 and 20 of 1RLG, which adopt the C3'-endo conformation. Our NMR data indicate that the conformation of these riboses is 
a

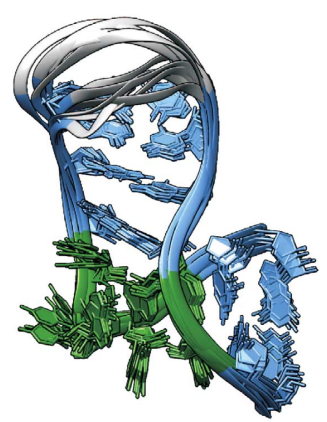

b

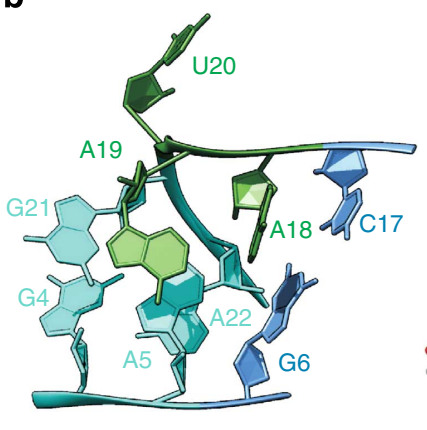

C

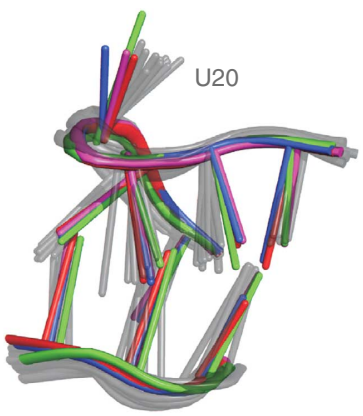

Figure 3 | ssNMR structure of the Pf Box C/D RNA. (a) Overlay of the 10 lowest energy structures of the Pf Box C/D RNA in complex with L7Ae from ssNMR data. Terminal nucleotides 1 and 25-26 are not shown. Colour code as in Fig. 1a. (b) k-turn of the Pf Box C/D RNA, showing the characteristic geometry. Internal loop, green; NC stem, cyan, C stem, light blue. (c) Comparison of the k-turn geometry of the Pf Box C/D RNA obtained by ssNMR (10 lowest energy structures, gray) with that of the crystallographic structure of the Af Box C/D RNA (PDB code 1RLG) ${ }^{7}$, red; Pf Box C/D RNA (PDB code $3 N M U)^{8}$, blue; Af Box C/D RNA (PDB code 4BWO) ${ }^{29}$, green; Ss Box C/D RNA (PDB code 3PLA) ${ }^{30}$, magenta.

\section{Table 1 | Structural statistics (20 structures out of 300 calculated, PDB code 2nOr).}

\section{NMR distance and dihedral constraints}

Distance restraints

Total distance restraints

Intra-residue

Inter-residue

Sequential $(|i-j|=1)$

Non-sequential ( $|i-j|>1)$

Hydrogen bonds

Total dihedral angle restraints

Glycosidic angle $\chi$

Sugar pucker

Backbone

Based on A-form geometry

\section{Structure statistics}

Violations (mean and s.d.)

Distance constraints ( $\AA$ )

Dihedral angle

constraints $\left(^{\circ}\right)$

Max. distance constraint

violation $(\AA)$

Max. dihedral angle

violation $\left(^{\circ}\right)$

Deviations from idealized geometry

Bond lengths $(\AA)$

Bond angles $\left({ }^{\circ}\right)$

Impropers $\left(^{\circ}\right)$

\subsection{8}

$0.461 \pm 0.002$

Average pairwise r.m.s.d. ( $\AA$ )

All RNA heavy

$0.319 \pm 0.001$

(2-9,14-24)

All RNA backbone

(2-9,14-24)

Kink-turn backbone

(4-6,17-22)
$0.0023 \pm 0.0001$

$0.64 \pm 0.02$

$0.41 \pm 0.02$

$0.9 \pm 0.2$

$1.0 \pm 0.2$

208

135

96

39
34

174

18

54

43

Solvent refined

$0.007 \pm 0.003$

$0.28 \pm 0.07$

0.24

3.4

$0.8 \pm 0.2$

$1.0 \pm 0.2$

$0.7 \pm 0.2$

$0.8 \pm 0.2$

C2'-endo, in agreement with the other three crystallographic structures. Similarly, the $\varepsilon$ and $\zeta$ angles of the same nucleotides of $1 \mathrm{RLG}$ deviate from the values of both our structures bundle and the other three crystallographic structures. The $\beta, \varepsilon$ and $\zeta$ torsion angles are not directly determined by any NMR parameter,

but rather restrained loosely by data base values (see Methods), ${ }^{31} \mathrm{P}-{ }^{13} \mathrm{C}$ and ${ }^{13} \mathrm{C}-{ }^{13} \mathrm{C}$ distances. Nevertheless, the distribution of these angles in the ssNMR bundle is quite narrow and in good agreement with the reference structures. The $\alpha$ and $\gamma$ torsion angles are the least well defined by the NMR distance restrains in the stretch 18-21 of the Box C sequence. Interestingly, high variability is observed for these torsion angles among the four reference structures as well, indicating that the k-turn geometry is tolerant to different values (Supplementary Fig. 10). The only clear discrepancy between the ssNMR structures bundle and the four references structures is observed for $\mathrm{A} 5-\alpha, \gamma$. The $\mathrm{A}^{2}{ }^{31} \mathrm{P}$ chemical shift value (Supplementary Table 1) does not allow to restrict the A5- $\alpha$ to the gauche \pm conformations ${ }^{31}$, as observed in the four reference structures. However, despite this local difference, the k-turn geometry of the ssNMR bundle agrees very well with that of the reference conformations, with an average backbone r.m.s.d. for k-turn nucleotides 4-6 and 17-22 of $1.3 \AA$ to the four crystallographic structures (Fig. 3c). Finally, the glycosidic torsion angles $\chi$ are determined from the intensities of the $\mathrm{C1}$ '-C8/C6 cross-peaks and nicely agree with those of the reference structures.

In summary, we demonstrate that the structure of RNA is accessible by ssNMR with excellent precision and accuracy, despite the difficulties caused by broad line widths and resonance overlap. We present a straightforward, manageable strategy that uses easy-to-produce nucleotide-type selective-labelled RNAs and sensitive magnetization transfer schemes. Our results make the folding of short RNAs and selectively labelled RNA stretches, as well as their interaction with proteins, accessible at high resolution in the context of large RNAs and RNP particles. We anticipate that our method will have a considerable impact in various fields of RNA processing and small RNA regulation (siRNA, miRNA, piRNA), where the dynamic nature of the molecular complexes represents an obstacle to crystallization.

\section{Methods}

Sample preparation. The L7Ae-Box C/D RNA complex was assembled from protein and RNA in 1:1 ratio and purified by size exclusion chromatography. L7Ae was expressed in Escherichia coli (LB medium) and purified over a Ni-Nta column. Nucleotide-type ${ }^{13} \mathrm{C},{ }^{15} \mathrm{~N}$ selective-labeled Box C/D RNA was prepared by in vitro transcription with T7 polymerase produced in house. Labeling patterns of the RNA were obtained using NTP mixtures where only one or two nucleotide types were either ${ }^{15} \mathrm{~N}$ or ${ }^{13} \mathrm{C}$, or double ${ }^{13} \mathrm{C},{ }^{15} \mathrm{~N}$ labeled.

Sequential resonance assignment and measurement of structural restraints used eight samples with different labeling patterns. Six samples consisted of double ${ }^{13} \mathrm{C},{ }^{15} \mathrm{~N}$ nucleotide-type selective-labelled RNAs: A,Clab-RNA, A,Glab-RNA, 
A,Ulab-RNA, C,Ulab-RNA, G, C ${ }^{\text {lab }}$-RNA and G, Ulab-RNA (Supplementary Fig. 1a-f); two samples contained single ${ }^{13} \mathrm{C}$ or ${ }^{15} \mathrm{~N}$ labelled nucleotide pairs: $\left(\mathrm{G}-{ }^{13} \mathrm{C}, \mathrm{A}^{-15} \mathrm{~N}\right){ }^{\text {lab }}-\mathrm{RNA}$ and $\left(\mathrm{G}^{13} \mathrm{C}, \mathrm{U}_{-}{ }^{15} \mathrm{~N}\right){ }^{\text {lab }}$-RNA (Supplementary Fig. 1g,h). Next to these RNAs, an additional RNA construct was used to facilitate assignment, where the GAAA tetra-loop is substituted with the UUCG tetra-loop.

The L7Ae-Box C/D RNA complex was concentrated to $20 \mathrm{mg} \mathrm{ml}^{-1}$ in buffer containing $25 \mathrm{mM}$ HEPES and $120 \mathrm{mM}$ sodium chloride at $\mathrm{pH} 7.5$, and subsequently mixed with equal amount of precipitation solution $(100 \mathrm{mM}$ sodium acetate, 30\% PEG 400 in $100 \mathrm{mM}$ HEPES, pH 7.5), as reported previously ${ }^{13,32,33}$. The sample was micro-crystallized by slow precipitation using a SpeedVac concentrator at room temperature for $\sim 2.5 \mathrm{~h}$. The complex precipitated at half volume. The precipitate was packed in the ssNMR rotor by centrifugation. The final sample contained $\sim 4 \mathrm{mg}$ of RNA and $6 \mathrm{mg}$ of L7Ae.

NMR spectroscopy. Solid-state NMR experiments were performed on a $700 \mathrm{MHz}$ SB Bruker Avance III spectrometer equipped with $3.2 \mathrm{~mm}$ MAS ${ }^{1} \mathrm{H} /{ }^{13} \mathrm{C} /{ }^{15} \mathrm{~N}$ probehead. ${ }^{13} \mathrm{C},{ }^{31} \mathrm{P}$ TEDOR experiments were acquired at $600 \mathrm{MHz}$ with a $\mathrm{WB}$ Bruker Avance III spectrometer equipped with a tunable ${ }^{1} \mathrm{H} / \mathrm{X} / \mathrm{Y}$ probehead at Bruker Biospin in Rheinstetten. The temperature of all experiments was $260 \mathrm{~K}$. ${ }^{13} \mathrm{C},{ }^{15} \mathrm{~N}$-TEDOR- ${ }^{13} \mathrm{C},{ }^{13} \mathrm{C}$-PDSD, ${ }^{13} \mathrm{C},{ }^{31} \mathrm{P}$-TEDOR, ${ }^{13} \mathrm{C},{ }^{15} \mathrm{~N}$-TEDOR and ${ }^{15} \mathrm{~N},{ }^{15} \mathrm{~N}$-RFDR experiments were performed at $16 \mathrm{kHz}$ MAS, while proton diffusion-based CHHC and NHHC experiments were performed at $13 \mathrm{kHz}$ MAS.

\section{${ }^{13} \mathbf{C}^{15} \mathrm{~N}$-TEDOR $-{ }^{13} \mathbf{C}_{\boldsymbol{1}}{ }^{13} \mathbf{C}$-PDSD. In the ${ }^{13} \mathrm{C},{ }^{15} \mathrm{~N}$-TEDOR $-{ }^{13} \mathrm{C},{ }^{13} \mathrm{C}$-PDSD} experiment (Supplementary Fig. $3 \mathrm{a}$ ) ${ }^{13} \mathrm{C}$ magnetization was prepared by standard ${ }^{1} \mathrm{H}-{ }^{13} \mathrm{C}$ cross polarization (mixing time, $200 \mu \mathrm{s}$ ). The ${ }^{13} \mathrm{C}-{ }^{15} \mathrm{~N}$ dipolar coupling was reintroduced in a short TEDOR mixing time $(1.5-2 \mathrm{~ms})$, during which magnetization was transferred to nearby ${ }^{15} \mathrm{~N}$ nuclei, and then, after $t_{1}$, back to the ${ }^{13} \mathrm{C}$. In $t_{1}$, we recorded the frequency of nitrogens close to carbons, as for example that of N1/N9 directly bound to $\mathrm{Cl}$ ' and $\mathrm{C} 8 / \mathrm{C} 6$. The following, long ${ }^{13} \mathrm{C},{ }^{13} \mathrm{C}$-PDSD step (mixing time, $200-700 \mathrm{~ms}$ ) transferred the ${ }^{15} \mathrm{~N}$-chemical shift labelled ${ }^{13} \mathrm{C}$ magnetization to nearby carbons. Finally, ${ }^{13} \mathrm{C}$ magnetization was detected during $t_{2}$. The ambiguity on the carbon from which the magnetization originates in the PDSD step, either $\mathrm{Cl}^{\prime}$ or $\mathrm{C} 8 / \mathrm{C} 6$, was lifted in three-dimensional experiments, where the ${ }^{13} \mathrm{C}$ frequency was recorded before the PDSD mixing. Alternatively, we evaluated the efficiency of the ${ }^{15} \mathrm{~N} 1 / \mathrm{N} 9-{ }^{13} \mathrm{C}$ transfer, which in several instances was found to be better towards the $\mathrm{C1}$ ' than towards C8/C6. As a third alternative, a ${ }^{13} \mathrm{C}$-band-selective-TEDOR transfer, with selectivity either on $\mathrm{Cl}$ ' or on $\mathrm{C} 6 / \mathrm{C} 8$, can be used to resolve the ambiguity. Cross-peaks were evaluated and translated into distance restraints. Distance ranges $(d)$ were applied for inter-nucleotide restraints as $3.5<d<9 \AA$, according to several previous studies ${ }^{34-36}$; the ranges for intranucleotide base-ribose restraints, $3<d<6 \AA$, and intra-nucleotide ribose-ribose restraints, $3<d<4 \AA$, were determined from the nucleotides' geometry.

${ }^{13} C_{\text {, }}{ }^{31}$ P-TEDOR. In the ${ }^{13} \mathrm{C},{ }^{31} \mathrm{P}$-TEDOR experiment (Supplementary Fig. 3b), after initial preparation of ${ }^{13} \mathrm{C}$ magnetization, the ${ }^{13} \mathrm{C},{ }^{31} \mathrm{P}$ dipolar coupling was reintroduced in a TEDOR mixing time of $3.2 \mathrm{~ms}$; the frequency of ${ }^{31} \mathrm{P}$ was monitored in $t_{1}$, while ${ }^{13} \mathrm{C}$ magnetization was detected during $t_{2}$. Optionally, a short ${ }^{13} \mathrm{C},{ }^{13} \mathrm{C}-$ PDSD step $(50-100 \mathrm{~ms})$ can be applied after TEDOR to transfer the ${ }^{13} \mathrm{C}$ magnetization to further carbon spins, such as $\mathrm{Cl}^{\prime}$. This experiment was useful to identify the ribose spin systems through the better-resolved $\mathrm{Cl}^{\prime}$ chemical shift. ${ }^{13} \mathrm{C}$, ${ }^{31} \mathrm{P}$ TEDOR spectra were recorded for $\mathrm{A}^{\text {lab }}$-RNA and G, $\mathrm{U}^{\text {lab }}$-RNA; due to the limited signal-to-noise, only one TEDOR mixing time was recorded $(3.2 \mathrm{~ms})$. The spectra yielded 17 non-trivial restraints, which were classified as $3<d<5 \AA$, as appropriate for a mixing time of $3.2 \mathrm{~ms}$.

${ }^{13} \mathrm{C}$-band-selective, ${ }^{15} \mathrm{~N}$-TEDOR. In the ${ }^{13} \mathrm{C}$-band-selective, ${ }^{15} \mathrm{~N}$-TEDOR experiment ${ }^{9}$ (Supplementary Fig. 3c), after initial preparation of ${ }^{13} \mathrm{C}$ magnetization, the ${ }^{13} \mathrm{C}-{ }^{31} \mathrm{~N}$ dipolar coupling was reintroduced in a TEDOR mixing time of $6-15 \mathrm{~ms}$ with band-selective ${ }^{13} \mathrm{C}$ inversion pulses; the long mixing allows transferring magnetization between carbons and nitrogens as far as $5-6 \AA$. The ${ }^{15} \mathrm{~N}$ and ${ }^{13} \mathrm{C}$ frequencies were recorded during $t_{1}$ and $t_{2}$, respectively. With a ${ }^{13} \mathrm{C},{ }^{15} \mathrm{~N}-\mathrm{TEDOR}$ that was selective for $\mathrm{Cl}^{\prime}$ and $\mathrm{C} 4$ ', we obtained four G-C1',C4'/A-N6,N9 crosspeaks from the $\left(\mathrm{G}^{-13} \mathrm{C}, \mathrm{A}-{ }^{15} \mathrm{~N}\right){ }^{\text {lab }}$-RNA and two G-C1'/U-N1,N3 cross-peaks from the $\left(\mathrm{G}^{-13} \mathrm{C}, \mathrm{U}-{ }^{15} \mathrm{~N}\right){ }^{\text {lab }}$-RNA. Also in this case, we did not acquire multiple TEDOR mixing times, due to limited signal-to-noise. Distance ranges $3<d<5 \AA$ and $3<d<7 \AA$ were attributed to the strong and weak peaks, respectively, at a mixing time of $12 \mathrm{~ms}$

${ }^{15} \mathbf{N},{ }^{15} \mathrm{~N}$-RFDR. In the ${ }^{15} \mathrm{~N},{ }^{15} \mathrm{~N}$-RFDR experiment (Supplementary Fig. $3 \mathrm{~d}$ ), ${ }^{15} \mathrm{~N}$ magnetization was prepared through a $300 \mu \mathrm{s}$ cross-polarization step and its frequency was recorded during $t_{1}$; subsequently, the magnetization was transferred to nearby nitrogen atoms via an RFDR mixing step of $20 \mathrm{~ms}$ and finally detected during $t_{2}$.

CHHC and NHHC. In the CHHC and NHHC proton spin diffusion-based experiments (Supplementary Fig. 3e,f, respectively), ${ }^{13} \mathrm{C}$ or ${ }^{15} \mathrm{~N}$ magnetization was prepared through a short cross-polarization mixing time of $100-200 \mu \mathrm{s}$, followed by $t_{1}$ evolution on either ${ }^{13} \mathrm{C}$ (CHHC) or ${ }^{15} \mathrm{~N}$ (NHHC). Next, the magnetization was transferred back to protons, from where, after a short proton mixing of 100$200 \mu$ s, it was transferred to nearby carbons with a $100-\mu$ s cross polarization step; finally the frequency of ${ }^{13} \mathrm{C}$ was recorded in $t_{2}$. Inter-proton distances $2<d<4$ and $2<d<5 \AA$ were attributed to the strong and weak signals, respectively, following previous studies ${ }^{19,27}$.

In all the experiments, protons were decoupled in the indirect and direct acquisition times using high-power SPINAL-64 (ref. 37) decoupling at $85-95 \mathrm{kHz}$ Chemical shifts were referenced as described by Morcombe and Zilm ${ }^{38}$. The spectra were processed with NMRPipe ${ }^{39}$ and visualized with NMRviewj ${ }^{40}$.

Structural calculation protocol. Structures were calculated using the Aria 1.2/ CNS 1.1 set-up $^{28,41}$ following a similar protocol as for structural calculations of RNA by solution-state NMR data ${ }^{11,42,43}$. Both canonical and non-canonical basepairs were incorporated in the structure calculation as distance restraints. Planarity was enforced through weak planarity restraints $\left(5 \mathrm{kcal} \mathrm{mol}^{-1} \AA^{-2}\right)$ for canonical base pairs and non-canonical base pair U3 - U23. Flexible planarity was introduced for the base pairs A5 . G21 and G4 - A22 by defining the plane that involves one atom of the acceptor and four atoms of the donor base to allow for propeller twist and tilt, as described in ref. 44.

The ribose conformation was restrained through the analysis of ribose chemical shifts $^{14}$ (Supplementary Fig. 2). The riboses of nucleotides G4, A5, A19, U20, G21 were given an S-type conformation, while the remaining nucleotides, except for G6 and A11-A13, were restrained to the N-type conformation. The dihedral angles $\alpha$, $\beta, \varepsilon$ and $\zeta$ were restrained to the range typical for A-form helix $\left(300^{\circ} \pm 30^{\circ}\right.$, $180^{\circ} \pm 30^{\circ},-135^{\circ} \pm 30^{\circ}$ and $300^{\circ} \pm 30^{\circ}$, respectively) for nucleotides $2,6-9$, $14-17,24$, which are involved in canonical base pairs; the $\alpha, \beta, \varepsilon$ and $\zeta$ angles of the remaining nucleotides were loosely restrained to the allowed ranges $\left(180^{\circ} \pm 150^{\circ}\right.$, $180^{\circ} \pm 110^{\circ},-125^{\circ} \pm 75^{\circ}$ and $180^{\circ} \pm 150^{\circ}$, respectively). Dihedral angles $\alpha$ and $\zeta$ of nucleotides G4, G6, A18, U20 and G21 were additionally restrained to $0^{\circ} \pm 120^{\circ}$ based on ${ }^{31} \mathrm{P}$ chemical shifts ${ }^{31}$. The dihedral angle $\gamma$ was restrained to the gauche + conformation for nucleotides involved in base pairs.

Three hundred structures were calculated in one iteration without the automated assignment or the distance calibration options of Aria 1.2 using an assigned distance list. Before minimization, we randomized all backbone dihedral angles. The minimization protocol used the force-field DNA-RNA-allatom-hj-opls.top and the following parameters in the four steps of simulated annealing (SA), together with the PROLSQ nonbonded parameters ${ }^{43}$ : (i) the SA protocol started with a hightemperature torsion angle simulated annealing phase of 100,000 steps at $20,000 \mathrm{~K}$ (time step of $22.5 \mathrm{fs}$ ); (ii) this was followed by a torsion angle dynamic cooling phase from 20,000 to $1,000 \mathrm{~K}$ in 100,000 steps and by two cartesian dynamic cooling phases with a time step of $2.5 \mathrm{fs}$ ((iii) from 2,000 to $1,000 \mathrm{~K}$ in 100,000 steps and (iv) from 1,000 to $50 \mathrm{~K}$ in 80,000 steps, respectively. Finally, 20 low energy structures were refined in water (TIP3P) with OPLS nonbonded parameters ${ }^{45}$. Standard ARIA force constants were used for the different restraint types (for example, distances $-50 \mathrm{kcal} \mathrm{mol}^{-1}$, and dihedrals $-200 \mathrm{kcal} \mathrm{mol}^{-1}$, in the final cooling step. $)^{28}$.

The final structures were analysed using $\mathrm{MolMol}^{46}$ and Chimera ${ }^{47}$. Figures were prepared with Chimera.

Analysis of the mutant Box C/D RNA with the UUCG tetra-loop. We measured two samples of the mutant Box C/D RNA containing the stable UUCG tetra-loop (UUCG-RNA) instead of the GAAA tetra-loop to aid and confirm the assignment. The $2 \mathrm{D}{ }^{13} \mathrm{C},{ }^{15} \mathrm{~N}$-TEDOR- ${ }^{13} \mathrm{C},{ }^{13} \mathrm{C}$-PDSD of the $(\mathrm{A}, \mathrm{U}){ }^{\mathrm{lab}}$-UUCG-RNA allowed identifying two adenosines of the GAAA tetra-loop of the wild-type RNA, which disappear in the mutant spectrum. In addition, we could confirm the assignment of A15, which does not overlap with any other spin-system in the UUCG-RNA. The ${ }^{2 D}{ }^{13} \mathrm{C},{ }^{15} \mathrm{~N}$-TEDOR- ${ }^{13} \mathrm{C},{ }^{13} \mathrm{C}$-PDSD spectrum of G, $\mathrm{C}^{\text {lab }}$-UUCG-RNA allowed the assignment of G10, whose spin-system is not present in the spectrum of the mutant RNA. In addition, the resonances of G14 shift slightly in the mutant with respect to the wild-type RNA, due to the different structure of the UUCG tetra-loop. This fact confirmed the assignment of the G14 spin-system.

\section{References}

1. Petkova, A. T. et al. A structural model for Alzheimer's beta-amyloid fibrils based on experimental constraints from solid state NMR. Proc. Natl Acad. Sci. USA 99, 16742-16747 (2002).

2. Jaroniec, C. P. et al. High-resolution molecular structure of a peptide in an amyloid fibril determined by magic angle spinning NMR spectroscopy. Proc. Natl Acad. Sci. USA 101, 711-716 (2004).

3. de Groot, H. J. M. Solid-state NMR spectroscopy applied to membrane proteins. Curr. Opin. Struct. Biol. 10, 593-600 (2000)

4. Opella, S. J. \& Marassi, F. M. Structure determination of membrane proteins by NMR spectroscopy. Chem. Rev. 104, 3587-3606 (2004)

5. Shi, L. C. et al. Conformation of a seven-helical transmembrane photosensor in the lipid environment. Angew. Chem. Int. Ed. 50, 1302-1305 (2011).

6. Marchanka, A. \& Carlomagno, T. Solid-state NMR and RNA structure: a new partnership? eMagRes 3, 119-128 (2014). 
7. Moore, T., Zhang, Y. M., Fenley, M. O. \& Li, H. Molecular basis of box C/D RNA-protein interactions: cocrystal structure of archaeal L7Ae and a box C/D RNA. Structure 12, 807-818 (2004)

8. Xue, S. et al. Structural basis for substrate placement by an archaeal Box C/D ribonucleoprotein particle. Mol. Cell 39, 939-949 (2010).

9. Cojocaru, V., Nottrott, S., Klement, R. \& Jovin, T. M. The snRNP 15.5K protein folds its cognate K-turn RNA: a combined theoretical and biochemical study. RNA 11, 197-209 (2005).

10. Goody, T. A., Melcher, S. E., Norman, D. G. \& Lilley, D. M. J. The kink-turn motif in RNA is dimorphic, and metal ion-dependent. RNA 10, 254-264 (2004).

11. Falb, M. et al. Structure of the K-turn U4 RNA: a combined NMR and SANS study. Nucleic Acids Res. 38, 6274-6285 (2010).

12. Schroeder, K. T., McPhee, S. A., Ouellet, J. \& Lilley, D. M. A structural database for k-turn motifs in RNA. RNA 16, 1463-1468 (2010).

13. Marchanka, A., Simon, B. \& Carlomagno, T. A suite of solid-state NMR experiments for RNA resonance assignment in a $21 \mathrm{kDa}$ protein-RNA complex. Angew. Chem. Int. Ed. 52, 9996-10001 (2013).

14. Cherepanov, A. V., Glaubitz, C. \& Schwalbe, H. High-resolution studies of uniformly C-13,N-15-labeled RNA by solid-state NMR spectroscopy. Angew. Chem. Int. Ed. 49, 4747-4750 (2010).

15. Jaroniec, C. P., Filip, C. \& Griffin, R. G. 3D TEDOR NMR experiments for the simultaneous measurement of multiple carbon-nitrogen distances in uniformly C-13, N-15-labeled solids. J. Am. Chem. Soc. 124, 10728-10742 (2002).

16. Szeverenyi, N. M., Sullivan, M. J. \& Maciel, G. E. Observation of spin exchange by two-dimensional Fourier-Transform C-13 cross polarization-magic-angle spinning. J. Magn. Reson. 47, 462-475 (1982).

17. Bennett, A. E., Ok, J. H., Griffin, R. G. \& Vega, S. Chemical-shift correlation spectroscopy in rotating solids-radio frequency-driven dipolar recoupling and longitudinal exchange. J. Chem. Phys. 96, 8624-8627 (1992).

18. Leppert, J. et al. Identification of NH...N hydrogen bonds by magic angle spinning solid state NMR in a double-stranded RNA associated with myotonic dystrophy. Nucleic Acids Res. 32, 1177-1183 (2004).

19. Lange, A., Luca, S. \& Baldus, M. Structural constraints from proton-mediated rare-spin correlation spectroscopy in rotating solids. J. Am. Chem. Soc. 124, 9704-9705 (2002).

20. Riedel, K. et al. Characterisation of hydrogen bonding networks in RNAs via magic angle spinning solid state NMR spectroscopy. J. Biomol. NMR 31, 331-336 (2005).

21. Farès, C., Amata, I. \& Carlomagno, T. C-13-Detection in RNA bases: revealing structure-chemical shift relationships. J. Am. Chem. Soc. 129, 15814-15823 (2007).

22. Ohlenschläger, O. et al. The structure of the stemloop D subdomain of coxsackievirus B3 cloverleaf RNA and its interaction with the proteinase 3C. Structure 12, 237-248 (2004).

23. Lewandowski, J. R., De Paëpe, G. \& Griffin, R. G. Proton assisted insensitive nuclei cross polarization. J. Am. Chem. Soc. 129, 728-729 (2007).

24. De Paëpe, G. et al. Proton assisted recoupling and protein structure determination. J. Chem. Phys. 129, 245101 (2008).

25. Loquet, A. et al. 3D structure determination of the Crh protein from highly ambiguous solid-state NMR restraints. J. Am. Chem. Soc. 130, 3579-3589 (2008).

26. Manolikas, T., Herrmann, T. \& Meier, B. H. Protein structure determination from C-13 spin-diffusion solid-state NMR spectroscopy. J. Am. Chem. Soc. 130, 3959-3966 (2008)

27. Lange, A. et al. A concept for rapid protein-structure determination by solidstate NMR spectroscopy. Angew. Chem. Int. Ed. 44, 2089-2092 (2005).

28. Linge, J. P., Habeck, M., Rieping, W. \& Nilges, M. ARIA: automated NOE assignment and NMR structure calculation. Bioinformatics 19, 315-316 (2003).

29. Huang, L. \& Lilley, D. M. The molecular recognition of kink-turn structure by the L7Ae class of proteins. RNA 19, 1703-1710 (2013).

30. Lin, J. et al. Structural basis for site-specific ribose methylation by box C/D RNA protein complexes. Nature 469, 559-563 (2011).

31. Varani, G., Aboul-ela, F. \& Allain, F. H. T. NMR investigation of RNA structure. Prog. Nucl. Magn. Reson. Spectrosc. 29, 51-127 (1996).

32. Jehle, S. et al. Intermolecular protein-RNA interactions revealed by $2 \mathrm{D}$ P-31-N15 magic angle spinning solid-state NMR spectroscopy. J. Am. Chem. Soc. 132, 3842-3846 (2010).
33. Asami, S., Rakwalska-Bange, M., Carlomagno, T. \& Reif, B. Protein-RNA interfaces probed by $1 \mathrm{H}$-detected MAS solid-state NMR spectroscopy. Angew. Chem. Int. Ed. 52, 2345-2349 (2013)

34. Wasmer, C. et al. Amyloid fibrils of the HET-s(218-289) prion form a beta solenoid with a triangular hydrophobic core. Science 319, 1523-1526 (2008).

35. Bertini, I. et al. High-resolution solid-state NMR structure of a $17.6 \mathrm{kDa}$ protein. J. Am. Chem. Soc. 132, 1032-1040 (2010).

36. Demers, J. P. et al. High-resolution structure of the Shigella type-III secretion needle by solid-state NMR and cryo-electron microscopy. Nat. Commun. 5, 4976 (2014).

37. Fung, B. M., Khitrin, A. K. \& Ermolaev, K. An improved broadband decoupling sequence for liquid crystals and solids. J. Magn. Reson. 142, 97-101 (2000).

38. Morcombe, C. R. \& Zilm, K. W. Chemical shift referencing in MAS solid state NMR. J. Magn. Reson. 162, 479-486 (2003).

39. Delaglio, F. et al. Nmrpipe-A multidimensional spectral processing system based on Unix pipes. J. Biomol. NMR 6, 277-293 (1995).

40. Johnson, B. A. \& Blevins, R. A. NMRView: a computer program for the visualization and analysis of NMR data. J. Biomol. NMR 4, 603-614 (1994).

41. Brunger, A. T. et al. Crystallography \& NMR system: a new software suite for macromolecular structure determination. Acta Crystallogr. Sect. D 54, 905-921 (1998).

42. Carlomagno, T. et al. Structural principles of RNA catalysis in a 2'-5' lariatforming ribozyme. J. Am. Chem. Soc. 135, 4403-4411 (2013).

43. Nozinovic, S. et al. High-resolution NMR structure of an RNA model system the 14-mer cUUCGg tetraloop hairpin RNA. Nucleic Acids Res. 38, 683-694 (2010).

44. Zanier, K. et al. Structure of the histone mRNA hairpin required for cell cycle regulation of histone gene expression. RNA 8, 29-46 (2002).

45. Linge, J. P. et al. Refinement of protein structures in explicit solvent. Proteins 50, 496-506 (2003).

46. Koradi, R., Billeter, M. \& Wüthrich, K. MOLMOL: a program for display and analysis of macromolecular structures. J. Mol. Graphics 14, 51-55 (1996).

47. Pettersen, E. F. et al. UCSF chimera-a visualization system for exploratory research and analysis. J. Comput. Chem. 25, 1605-1612 (2004).

\section{Acknowledgements}

This work has been supported by the BMBF through grant $0315870 \mathrm{~B}$ to T.C.

\section{Author contributions}

A.M. produced samples, developed and performed both NMR experiments and structure calculations, interpreted the results and wrote the manuscript together with T.C. B.S contributed to the development of pulse programs and structural calculation protocols. G.A. helped in the acquisition of NMR data. T.C. designed the project, contributed to the development of pulse programs and structural calculation protocols, interpreted the results and wrote the manuscript together with A.M. All the authors discussed the results reported in the manuscript.

\section{Additional information}

Accession codes: The atomic coordinates of 10 lowest energy structures have been deposited in the Protein Data Bank under accession number 2N0R. The NMR chemical shifts have been deposited in the Biological Magnetic Resonance Data Bank, entry 25534

Supplementary Information accompanies this paper at http://www.nature.com/ naturecommunications

Competing financial interests: The authors declare no competing financial interests.

Reprints and permission information is available online at http://npg.nature.com/ reprintsandpermissions/

How to cite this article: Marchanka, A. et al. RNA structure determination by solid-state NMR spectroscopy. Nat. Commun. 6:7024 doi: 10.1038/ncomms8024 (2015).

This work is licensed under a Creative Commons Attribution 4.0 International License. The images or other third party material in this article are included in the article's Creative Commons license, unless indicated otherwise in the credit line; if the material is not included under the Creative Commons license, users will need to obtain permission from the license holder to reproduce the material. To view a copy of this license, visit http://creativecommons.org/licenses/by/4.0/ 